\title{
Optical response of strongly absorbing inhomogeneous materials: Application to paper degradation
}

\author{
M. Missori, ${ }^{1}$ O. Pulci, ${ }^{2,3}$ L. Teodonio, ${ }^{1,4}$ C. Violante, ${ }^{2}$ I. Kupchak, ${ }^{5}$ J. Bagniuk, ${ }^{6}$ J. Łojewska, ${ }^{6}$ and A. Mosca Conte ${ }^{2}$ \\ ${ }^{1}$ Istituto dei Sistemi Complessi, Consiglio Nazionale delle Ricerche, Via Salaria Km 29.300, 00015 Monterotondo Scalo (Rome), Italy \\ ${ }^{2}$ ETSF, MIFP, Dipartimento di Fisica, Università di Roma Tor Vergata, Via della Ricerca Scientifica 1, I-00133 Rome, Italy \\ ${ }^{3}$ Istituto di Struttura della Materia, Consiglio Nazionale delle Ricerche, Via del Fosso del Cavaliere 100, I-00133 Rome, Italy \\ ${ }^{4}$ Centro Italiano di Studi e Ricerche di Storia e Tecnologia della Carta “Andrea F. Gasparinetti,” Via M. Bellocchi 20, \\ 60044 Fabriano (AN), Italy \\ ${ }^{5}$ MIFP, V. Lashkarev Institute of Semiconductor Physics of National Academy of Sciences of Ukraine, pr. Nauki 45, 03680, Kiev, Ukraine \\ ${ }^{6}$ Faculty of Chemistry, Jagiellonian University, Ingardena 3, 30-060 Kraków, Poland
}

(Received 14 November 2013; revised manuscript received 14 January 2014; published 6 February 2014)

\begin{abstract}
In this paper, we present a new noninvasive and nondestructive approach to recover scattering and absorption coefficients from reflectance measurements of highly absorbing and optically inhomogeneous media. Our approach is based on the Yang and Miklavcic theoretical model of light propagation through turbid media, which is a generalization of the Kubelka-Munk theory, extended to accommodate optically thick samples. We show its applications to paper, a material primarily composed of a web of fibers of cellulose, whose optical properties are strongly governed by light scattering effects. Samples studied were ancient and industrial paper sheets, aged in different conditions and highly absorbing in the ultraviolet region. The recovered experimental absorptions of cellulose fibers have been compared to theoretical ab initio quantum-mechanical computational simulations carried out within time-dependent density functional theory. In this way, for each sample, we evaluate the absolute concentration of different kinds of oxidized groups formed upon aging and acting as chromophores causing paper discoloration. We found that the relative concentration of different chromophores in cellulose fibers depends on the aging temperature endured by samples. This clearly indicates that the oxidation of cellulose follows temperature-dependent reaction pathways. Our approach has a wide range of applications for cellulose-based materials, like paper, textiles, and other manufactured products of great industrial and cultural interest, and can potentially be extended to other strongly absorbing inhomogeneous materials.
\end{abstract}

DOI: 10.1103/PhysRevB.89.054201

PACS number(s): 71.15.Mb, 81.70.Fy, 78.20.Ci

\section{INTRODUCTION}

Optically inhomogeneous materials are a wide class of materials characterized by the presence of light scattering, which affects their optical response. They play an important role in several research fields ranging from the remote characterization of planetary atmospheres [1] and surfaces [2], to medicine and biology [3], to the study of rough surfaces provided by energy collectors [4] up to cultural heritage such as paintings [5,6] and ancient paper [7,8].

A medium is optically inhomogeneous when it is characterized by variations of the dielectric function $\varepsilon$ over distances comparable to those of the wavelength of light impinging on the sample. This induces the scattering of light, namely the deflection of photons from a straight path [9]. As a consequence, the usual laws related to optical properties of homogeneous materials are no more valid [10]. Practically, this can be caused by, for example, irregularities in the propagation medium, presence of particles, or roughness in the interface between two media. In the presence of scattering, the reflectivity of materials is commonly referred to as diffuse reflectance [10].

In this context, paper sheets represent a striking example of materials whose optical properties are strongly governed by light scattering effects. Paper sheets are complex systems primarily composed of a web of fibers of cellulose whose diameter ranges from about 1 to about $10 \mu \mathrm{m}[11,12]$. The white appearance of nondegraded paper is due to its light scattering effect due to the presence of the smaller components arranged in irregular ways [15]. Paper is a material of great relevance both for its industrial applications and for its historical value. It is largely employed in several applications, the most common of which is as a writing support. In the industrial field, it is also used as an insulator in high-power electrical transformers. In ancient times, paper was derived from pure cellulose fibers of cotton, linen, or hemp. Currently, it is mostly obtained from wood pulp. Paper can also contain additives, mainly sizing and fillers (such as starch, gelatine, rosin, alum, chalk), depending on the production technique employed $[13,14]$.

Cellulose is a linear homopolymer composed of $\beta$-Dglucopyranose units $\left(\mathrm{C}_{6} \mathrm{H}_{10} \mathrm{O}_{5}\right)_{n}$, linked together up to form chains with $n$ from $\approx 10^{2}$ to $\approx 10^{4}$. Fibers are formed because cellulose chains have a strong tendency to aggregate into highly ordered structural entities through an extended network of both intra and intermolecular hydrogen bonds. As a consequence, a hierarchical arrangement is formed from single chains up to fibers. The basic components of the cellulose supramolecular structure (elemental fibrils) include assembly of highly ordered (crystalline) domains and of disordered (amorphous-like) regions [16].

From a microscopic perspective, paper degradation can be seen as the combination of three most important processes: (i) recrystallization, (ii) acid hydrolysis, and (iii) oxidation of the $\beta$-D-glucopyranose units, with the subsequent development of various chemical products $[8,16-19]$. The degradation extent in paper after a period of time depends on the 
environmental conditions to which it has been subjected $[8,18]$. Among the oxidation products, those that are responsible for yellowing are called chromophores. Pristine cellulose does not absorb light above about $200 \mathrm{~nm}$ (below $6 \mathrm{eV}$ ) [8,18]. The yellow color seen in aged papers is mainly due to the fact that chromophores in paper absorb the higher energy band of visible light (corresponding to violet and blue) and largely scatter the yellow and red portion, thereby producing the characteristic yellow-brown hue $[8,15,20,21]$.

To separate the effect of the scattering from the absorption on the spectroscopic composition of the radiation flux reflected or transmitted by a layer, a number of theories have been developed [10]. Among those, some phenomenological models have been applied to describe paper optical properties [10,22,23]. However, the most successful approach is the Kubelka-Munk (K-M) theory [22], a two-flux approach to the general radiation transfer theory, describing the propagation through a medium that absorbs, emits, and scatters light. Recently, the K-M model has been extended by Yang and Miklavcic (Y-M) to accommodate a wider range of optical absorption [24,25].

In this paper, we present a new noninvasive and nondestructive approach for recovering scattering and absorption coefficients from reflectance measurements of highly absorbing optically inhomogeneous media. We introduce an innovative advance to the Yang and Miklavcic theoretical model to manage optically thick samples. In this way, the optical properties of paper were investigated up to the ultraviolet region where degraded paper sheets show strong optical absorption. The experimental absorption coefficients of oxidized cellulose fibers have been compared to theoretical $a b$ initio quantummechanical computational simulations carried out within timedependent density functional theory (TDDFT). Accordingly, we evaluate the absolute concentration of different kinds of oxidized groups induced by aging and acting as chromophores causing paper discoloration. In this way, chemical information was extracted at the molecular level following nondestructive and noninvasive optical reflectance measurements.

\section{MATERIALS AND EXPERIMENTAL METHODS}

\section{A. Materials \\ 1. Ancient samples}

The ancient sample named 38_C3 is dated back from 1466 to 1529 and originates from the city of Strozza (Bergamo, Italy). Its thickness is $100 \pm 10 \mu \mathrm{m}$. The sample named 70_L15 is dated back from 1441 to 1446 and originates from Pula (Croatia). Its thickness is $130 \pm 10 \mu \mathrm{m}$. Both samples belong to the Corpus Chartarum Italicarum (CCI), a collection of about 5000 watermarked papers conserved at the Istituto Centrale per il Restauro e la Conservazione del Patrimonio Archivistico e Librario (Central Institute for the Restoration of Archival and Library Heritage) in Rome, Italy. The two ancient samples show different degrees of degradation and discoloration. In accordance with the custom in ancient times, these samples are made of cotton and linen cellulose fibers. Both ancient samples carried no printing.

\section{Transformers samples}

The transformer sample named TA originates from Energopomiar Elektryka company (Poland). This sample was taken from insulation of higher voltage winding in a power transformer after its failure. At the origin, sample TA was embedded with mineral oil, normally used to improve electrical insulation in power transformers. To allow optical analysis of paper substrate, the mineral oil was removed by using several baths of toluene, each lasting few minutes with mild beating. Then, residues of toluene were evaporated under low pressure. Complete removal of mineral oil was checked by Fourier transform infrared spectroscopy. At the same time, this technique allowed to evidence that sample TA was made of pure cellulose paper (without lignin and fillers). The thickness of the sample is $50 \pm 10 \mu \mathrm{m}$.

\section{B. Experimental methods}

\section{Volume fraction of cellulose fibers}

The thickness $t$ of the paper sheets was measured using a paper-gauging micrometer (with a sensitivity of $10 \mu \mathrm{m}$ ) in several locations and averaging the results. The mass $m$ of the sheet was measured using a laboratory scale. Once $t$, $m$, and the sheets surface area $\Sigma$ are known, it is possible to estimate the paper's density by $\rho=\frac{m}{\Sigma t}$. The value of $\rho$ can be compared to the average density of cellulose fibers $\rho_{c}=1.5 \mathrm{~g} / \mathrm{cm}^{3}$. Since paper is basically composed of fibers and voids, the ratio $\frac{\rho}{\rho_{c}}$ indicates the volume fraction $v$ of cellulose fibers in the paper sheets, a value required for chromophore concentration calculations. The values of $v$ are 0.475 for sample $70 \_15,0.604$ for sample 38_C3, and 0.552 for sample TA.

\section{Diffuse reflectance measurements}

The absolute diffuse reflectance $(R)$ spectra in the ultraviolet (UV), visible (Vis), and near infrared (NIR) regions were measured by a setup from Avantes BV (The Netherlands). The UV-Vis-NIR source was a combined deuterium-halogen AvaLight-DH-S-BAL light source. This was connected by a 800- $\mu$ m-diameter optical fiber to a 30-mm-diameter Spectralon coated integrating sphere (model AvaSphere-30-REFL), used to illuminate the sample with diffuse radiation and collect radiation reflected from the paper surface over all angles. The integrating sphere was laid on the specimens and its sample port was $6 \mathrm{~mm}$ in diameter. Radiation from the integrating sphere was sent by a $600-\mu \mathrm{m}$-diameter optical fiber to an AvaSpec-2048x14-USB2 spectrometer equipped with a 2048x 14 CCD detector. The spectrometer used a diffraction grating allowing applications in the 248-1050-nm range (photon energy from 1.18 to $4.96 \mathrm{eV}$ ) with a 2.4-nm spectral resolution. A personal computer was used for spectrometer control and data recording.

For each measured circular area on the samples, two sets of spectra were measured, obtained by laying the sheets on a white $\left(R_{w}\right)$ and then a black $\left(R_{b}\right)$ backing [10,15,24-26]. The reflectances of the backings, $R_{w b}$ and $R_{b b}$ for white and black, respectively, were measured separately. All measured spectra were normalized to a Spectralon diffuse reflectance reference standard (factory calibrated) allowing the absolute reflectance to be recovered. With this setup, by averaging the signals of ten consecutive acquisitions lasting three seconds each, we were 
able to acquire $R$ spectra of the paper samples with a absolute error of about $2 \times 10^{-3}$.

\section{Absorption coefficient of optically thick samples}

The experimental intrinsic absorption coefficient $\alpha$ of cellulose fibers in the paper samples was recovered from the $R$ spectra by extending the Y-M theoretical model describing light propagating through turbid media [24,25]. The model is a generalization of the revised K-M theory [10,26], extending its applicability to accommodate a wider range of absorption influences.

In the Y-M approach, the effect of photon scattering on path length of light propagating into an absorbing and scattering medium is taken into account. This leads to new analytical relationships between the phenomenological scattering $S$ and absorption $K \mathrm{~K}-\mathrm{M}$ coefficients and the intrinsic scattering $s$ and absorption $\alpha$ coefficients of a material.

As a first step, from $R_{w}, R_{b}, R_{w b}$, and $R_{b b}$, using the results of the original K-M theory [10], the reflectance $R_{\infty}$ that would have an infinite layer of the same sample can be calculated:

$$
R_{\infty}=a-\sqrt{a^{2}-1}
$$

where

$$
a=\frac{\left(R_{w b}-R_{b b}\right)\left(1+R_{w} R_{b}\right)-\left(R_{w}-R_{b}\right)\left(1+R_{w b} R_{b b}\right)}{2\left(R_{b} R_{w b}-R_{w} R_{b b}\right)} .
$$

Results for $R_{\infty}$ as well as for measured $R_{w}$ and $R_{b}$ for each paper sample are shown in Fig. 1. For each sample, in the NIR and Vis range, $R_{w}$ shows higher values with respect to $R_{\infty}$, which, in turn, is higher than $R_{b}$; for ancient samples (38_C3 and 70_L15), this behavior is true up to a certain energy from which the three spectra become indistinguishable within the measurement error. This is due the increasing absorption of paper in the higher-energy side of the visible spectrum and in the UV due to the presence of chromophores in the cellulose fibers and to the relatively higher values of $t$ of

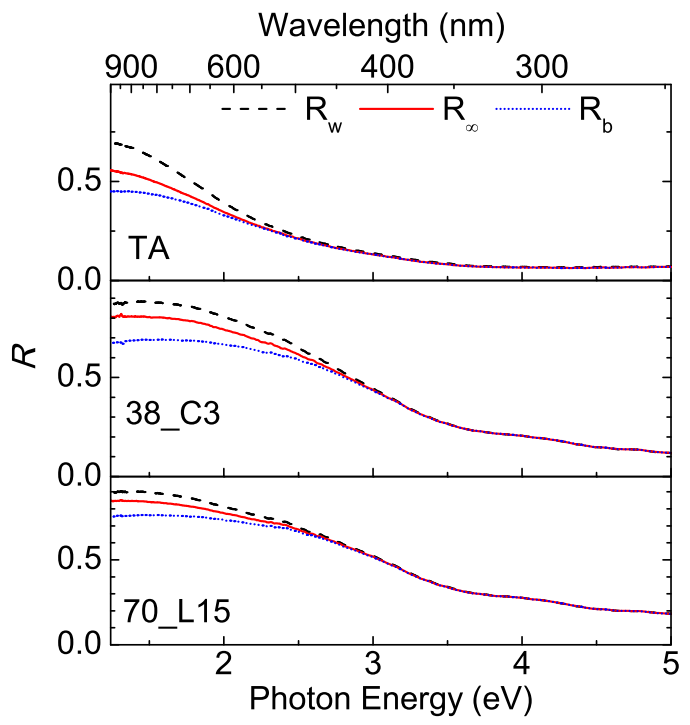

FIG. 1. (Color online) Measured $R_{w}, R_{b}$, and corresponding $R_{\infty}$ as calculated from Eqs. (1) and (2) for the paper samples. these samples [8]. For sample TA, instead, mainly because of its lower value of $t$, the three spectra remain distinguishable within all the measured range (even if above $2.5 \mathrm{eV}$ their differences approach the experimental error on $R$ ).

Once $R_{\infty}$ is determined, it is possible to recover the $S$ and $K$ coefficients:

$$
\begin{gathered}
S=\frac{1}{t\left(\frac{1}{R_{\infty}}-R_{\infty}\right)} \ln \frac{\left(1-R_{b} R_{\infty}\right)\left(R_{\infty}-R_{b b}\right)}{\left(1-R_{\infty} R_{b b}\right)\left(R_{\infty}-R_{b}\right)}, \\
K=\frac{S\left(1-R_{\infty}\right)^{2}}{2 R_{\infty}} .
\end{gathered}
$$

From the knowledge of $S$ and $K$ and by using the Y-M equations [24,25] for diffuse light distribution, the intrinsic absorption $\alpha$ and scattering $s$ coefficients of paper can be recovered:

$$
\begin{aligned}
\alpha & =\frac{K}{2 \mu}, \\
s & =\frac{S}{\mu},
\end{aligned}
$$

where

$$
\begin{gathered}
\mu=\sqrt{2 S D}, \\
D=\frac{1}{A} \frac{1-2 A t e^{(-A t)}-e^{(-2 A t)}}{1-2 e^{(-A t)}+e^{(-2 A t)}},
\end{gathered}
$$

and

$$
A=\sqrt{K^{2}+2 K S},
$$

where all variables depend on the wavelength $\lambda$ of incident radiation. The quantity $\mu=\mu(s, \alpha)$ is the scattering-induced path variation (SIPV) factor [24,25]. It describes the influence of light scattering on the total path length and is nonlinearly dependent on both the absorption and scattering properties of the medium.

$D$ represents the average depth of photons that undergo reflection and exit the upper surface of the paper sheet [24,25]. In Fig. 2, D is reported for the samples studied

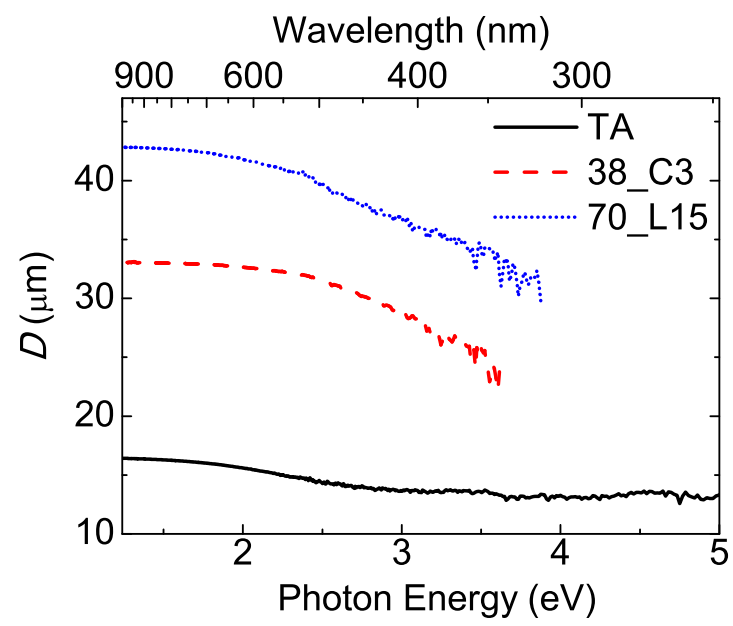

FIG. 2. (Color online) The average depth $D$ of photons that undergo reflection and exit the upper surface of the paper samples as function of wavelength. Note that $D \ll t$ for each sample and it further decreases for shorter wavelengths. 
in the energy range where $R_{w} \neq R_{b}$. As the wavelength decreases, $D$ decreases due to the increasing optical absorption of chromophores in cellulose fibers. For sample TA, instead, $D$ is defined over all the measured energy range, and it decreases from 16.5 to $13 \mu \mathrm{m}$. For sample 38 C $3, D$ is defined up to about $3.6 \mathrm{eV}$ (344 nm) and it decreases from 33 to $23 \mu \mathrm{m}$. For sample 70_L15, $D$ is defined up to about $3.9 \mathrm{eV}(320 \mathrm{~nm})$ and it decreases from 43 to $31 \mu \mathrm{m}$. These values, if compared to the much higher values of thickness $t$ of the sheets $(50,100$, and $130 \mu \mathrm{m}$ for samples TA, 38_C3, and 70_L15, respectively) explain the small (sample TA) or negligible (ancient samples) effect of different backings at shorter wavelength.

If $R_{w}$ and $R_{b}$ assume the same value, singularities in the K-M equation for $S$ [Eq. (3)] appear, because of the vanishing denominator in the argument of the logarithm $\left(R_{\infty}=R_{b}\right)$. On the other hand, $R_{\infty}$ and $a$ can anyhow be calculated even in this case since their expressions [Eqs. (1) and (2)] do not result in singularities. Therefore, since $K$ depends only on $S$ and $R_{\infty}$ [Eq. (4)], in order to estimate $K$, a different approach to estimate $S$ in the wavelength region where samples are optically thick is needed. Once $K$ has been recovered, $\alpha$ can be calculated from Eq. (5).

With this aim in mind, let us recall that the portion of radiation scattered throughout the solid angle by a layer of an inhomogeneous medium, in a given time interval, can be expressed as [12,27]

$$
s_{\infty}(\lambda) \propto z(\lambda) T(\lambda) \sigma(\lambda),
$$

where $z$ is the penetration depth of photons and $T$ is the layer transmission coefficient for the incident radiation of wavelength $\lambda . s_{\infty}$ of Eq. (10) has the same physical meaning of $s$, the intrinsic scattering coefficient of the medium of the $\mathrm{K}-\mathrm{M}$ theory, and its physical dimensions are the inverse of a length. $s_{\infty}$ represents the scattering coefficient estimated for higher photon energy (smaller wavelengths) where the paper sheet is optically thick and Eq. (3) diverges.

The total scattering cross-section $\sigma(\lambda)$ per unit volume of a collection of scatterers depends on their geometrical and physical parameters such as their number per unit volume, their volume, and the refractive index difference with respect to the surrounding medium (contrast) [27]. For paper, scatterers are mainly cellulose fibers (and, if present, pigments and mineral fillers)

The dependence of $\sigma(\lambda)$ on $\lambda$ can be approximated as $\lambda^{-4}$ in the Rayleigh scattering regime where scatterer sizes are smaller than $\lambda$, or as $\lambda^{-k}$, with $0<k<2$, in the Mie scattering approximation for scatterer sizes comparable to $\lambda$ [28]. For a material like paper, which is made of a collection of not uniformly sized rough-surfaced fibers, the scattering efficiency varies smoothly with the wavelength. This is well demonstrated in Fig. 3, where $s$ shows an almost constant trend in energy $(\lambda)$ for the range where absorption is negligible. Since the penetration depth $z$ and the sample transmission $T$ are constant in $\lambda$ in this range, $\sigma(\lambda)$ is also constant. Therefore, in the following calculations, we consider the total scattering cross-section of the paper sheets constant within the spectral region considered in this study.

In order to estimate the dependence of $s_{\infty}$ on $\lambda$ in the wavelength range where paper shows a strong optical

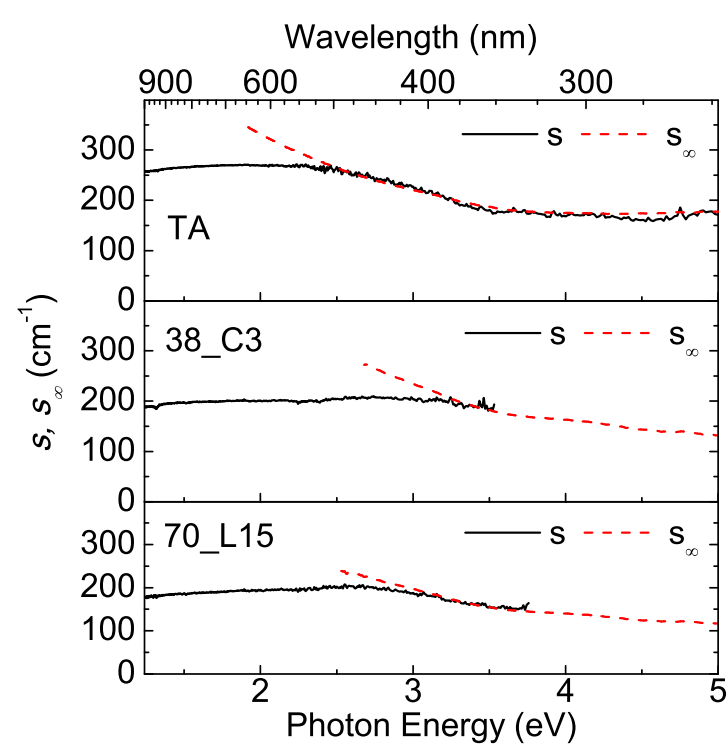

FIG. 3. (Color online) $s$ and $s_{\infty}$ are shown for the samples measured as a function of energy (wavelength).

absorption, let us calculate the dependence on $\lambda$ of the product $z T$ appearing in Eq. (10).

Since $D$ represents the average depth of photons that undergo reflection and exit the upper surface of the paper sheet, we can approximate the penetration depth $z$ in Eq. (10) with $D$. When paper is optically thick, i.e., $A t \gg 1$ in Eq. (8), it can readily be deduced that the explicit dependence on $t$ disappears and that, to a good approximation [24,25],

$$
D \approx D_{\infty}=\frac{1}{A}=\frac{1}{\sqrt{K^{2}+2 K S}}=\frac{1}{K \sqrt{\left(1+\frac{2}{A_{\mathrm{KM}}}\right)}},
$$

where we have introduced $A_{\mathrm{KM}}$ from Eq. (4),

$$
A_{\mathrm{KM}}=\frac{K}{S}=\frac{\left(1-R_{\infty}\right)^{2}}{2 R_{\infty}},
$$

the so-called Kubelka-Munk pseudoabsorption or remission function [10], which can be readily recovered from the experimental spectra once $R_{\infty}$ is calculated from Eqs. (1) and (2).

For $K>S$ [which, from Eqs. (5) and (6), is equivalent to $\alpha>s / 2$ ], the sample transmission coefficient $T$ can be expressed as [10]

$$
T \approx e^{-K z} \approx e^{-K D_{\infty}} \approx e^{-\frac{1}{\sqrt{1+\frac{2}{A_{\mathrm{KM}}}}}}
$$

having approximated $z$ with the $D_{\infty}$. Therefore

$$
\begin{aligned}
s_{\infty} \propto z T & \approx D_{\infty} e^{-K D_{\infty}} \\
& =\frac{1}{K \sqrt{1+\frac{2}{A_{\mathrm{KM}}}}} e^{-\frac{1}{\sqrt{1+\frac{2}{A_{\mathrm{KM}}}}}} .
\end{aligned}
$$

Remembering from Eqs. (5), (6), and (12) that $K$ depends on $s$, let us express $K$ as $K=s \mu A_{\mathrm{KM}} \approx s_{\infty} \mu_{\infty} A_{\mathrm{KM}}$. 
When paper is optically thick, the SIPV factor $\mu$ can be expressed in terms of $A_{\mathrm{KM}}$ from Eqs. (5)-(7) [24,25]:

$$
\mu_{\infty}=\sqrt{2 S D_{\infty}}=\sqrt{\frac{2 S}{\sqrt{K^{2}+2 K S}}}=\frac{1}{\left(\frac{A_{\mathrm{KM}}^{2}}{4}+\frac{A_{\mathrm{KM}}}{2}\right)^{\frac{1}{4}}} .
$$

Accordingly, Eq. (14) can be finally written as

$$
\begin{aligned}
& s_{\infty} \propto \sqrt{\frac{e^{-\frac{1}{\sqrt{1+\frac{2}{A_{\mathrm{KM}}}}}}}{\mu_{\infty} A_{\mathrm{KM}} \sqrt{1+\frac{2}{A_{\mathrm{KM}}}}}} \\
& \approx \sqrt{\frac{e^{-\frac{1}{\sqrt{1+\frac{2}{A_{\mathrm{KM}}}}}}}{\frac{1}{\left(\frac{A_{\mathrm{KM}}^{2}}{4}+\frac{A_{\mathrm{KM}}}{2}\right)^{\frac{1}{4}}} A_{\mathrm{KM}} \sqrt{1+\frac{2}{A_{\mathrm{KM}}}}}} \\
& =\sqrt{\frac{e^{-\frac{1}{\sqrt{1+\frac{2}{A_{\mathrm{KM}}}}}}}{\left[4\left(A_{\mathrm{KM}}^{2}+2 A_{\mathrm{KM}}\right)\right]^{\frac{1}{4}}},}
\end{aligned}
$$

an expression that can be easily calculated from the measured $R$ spectra even in case of optically thick samples. This equation is the principal result of this paper for what concerns the extension of the experimental measurements of the optical properties of paper to the ultraviolet region.

In Fig. 3,s and $s_{\infty}$ are shown as a function of photon energy and $\lambda$. In particular, since $s$ [from Eqs. (3) and (6)] for sample TA is defined in all the measured range, it is possible to verify the goodness of its estimation by $s_{\infty} . s_{\infty}$ agrees smoothly with $s$ when the condition $\alpha>s / 2$ starts to be valid. The availability of the intrinsic scattering coefficient of paper samples at any $\lambda$ in the measured range allows us to extract the intrinsic absorption coefficient $\alpha(\lambda)$ for comparison with theoretical calculations.

\section{THEORETICAL ANALYSIS}

The experimental optical absorption spectra have been compared to those simulated by using a TDDFT-based method [29]. TDDFT is a powerful approach to compute optical spectra of complex systems (see, for example, Refs. [30,31]). In order to simulate the optical properties of paper, we considered an infinitely extended crystal of cellulose. The unitary cell is composed of 42 atoms forming two $\beta$-D-glucopyranose units bonded by glycosidic links in the crystallographic phase called cellulose- $\mathrm{I}_{\beta}[32,33]$. The crystal is monoclinic with Bravais vectors taken from x-ray diffraction data [32]: (19.97, $0.0,0.0),(0.0,16.13,0.0)$, and $(0.0,8.86,7.59)$ expressed in Bohr. The atomic coordinates inside the cell were calculated within density functional theory (DFT) [34] with a BLYP [35] exchange-correlation functional. These calculations were carried out by using the plane-wave DFT code QUANTUM ESPRESSO [36]. Aged paper was simulated by including an oxidized group in the structure of pristine cellulose. For each group, we calculated the geometry of the system by minimizing the total energy with respect to atoms displacements. Then, we calculated the macroscopic dielectric function of cellulose by using the Casida algorithm [37] within TDDFT adiabatic
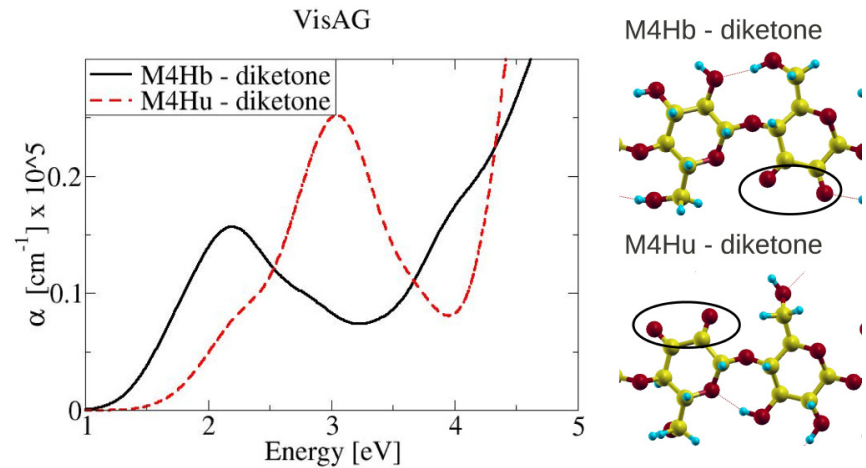

$\mathrm{M} 4 \mathrm{Hu}$ - diketone

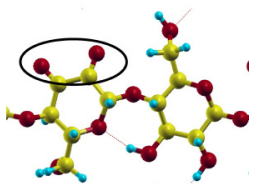

FIG. 4. (Color online) Relaxed structure and theoretical TDDFT absorption of VisAG. Yellow, red, and small blue spheres represent, respectively, carbon, oxygen, and hydrogen atoms.

local density approximation (ALDA) [38] and, finally, the theoretical absorption:

$$
\alpha=\frac{4 \pi}{\lambda} \sqrt{\frac{\sqrt{\varepsilon_{1}^{2}+\varepsilon_{2}^{2}}-\varepsilon_{1}}{2}},
$$

where $\varepsilon_{1}$ and $\varepsilon_{2}$ are, respectively, the real and the imaginary parts of the dielectric function $\varepsilon=\varepsilon_{1}+i \varepsilon_{2}$.

In our model, we only considered the oxidized groups showing optical absorption below $4.96 \mathrm{eV}$. We therefore selected eight oxidized groups that are displayed in Figs. 4-6 together with their absorption spectra. They were grouped into three different categories according to the energy range of their optical absorption spectrum. The visible absorbing groups (VisAG) contain diketones absorbing in all the visible spectrum and in the UV range. The low-UV absorbing groups (LUVAG) contain ketones strongly bonded by intrachain hydrogen bonds and aldheydes. These oxidized groups absorb in the UV range and in the blue-violet region. Finally, the UV absorbing groups (UVAG) do not absorb in the visible range but only in UV region and contain all the other ketones.

TDDFT calculations have been performed by using the DPFOREXC code [39]. Theoretical spectra of aged cellulose are calculated following Refs. [8,20,21], as linear combinations of these spectra computed with one oxidized group per
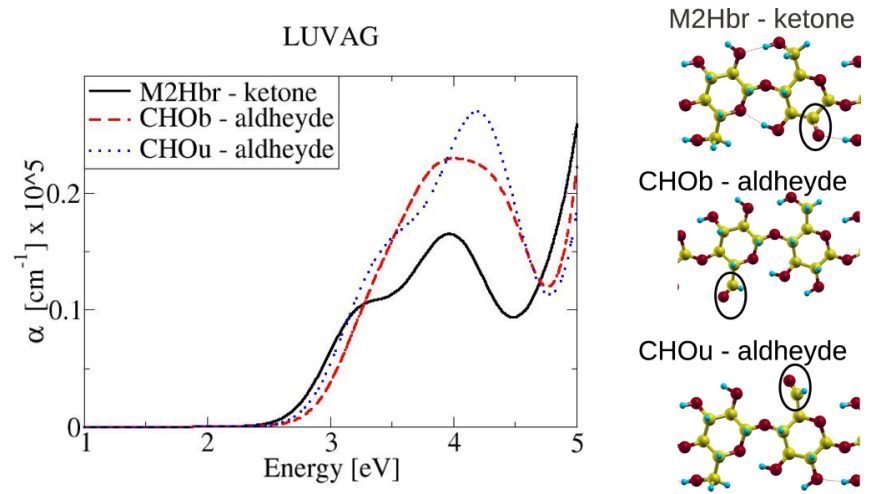

FIG. 5. (Color online) Relaxed structure and theoretical TDDFT absorption of LUVAG. Yellow, red, and small blue spheres represent, respectively, carbon, oxygen, and hydrogen atoms. 

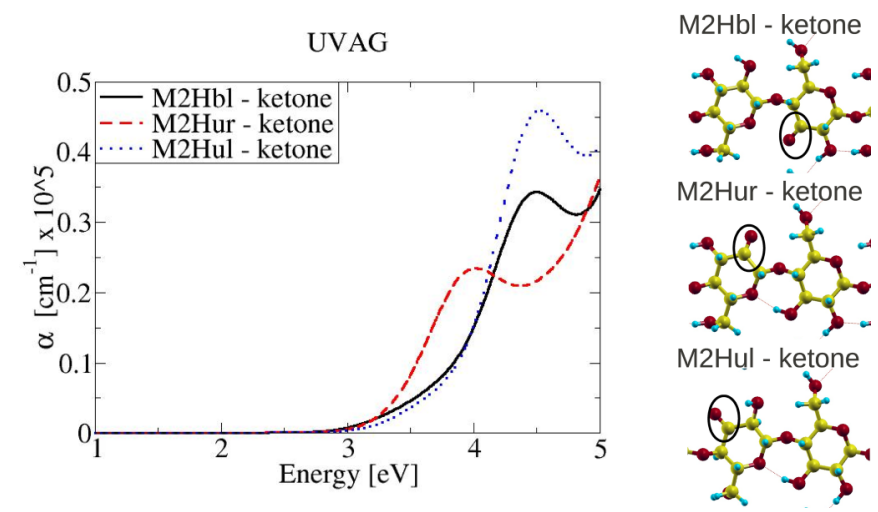

FIG. 6. (Color online) Relaxed structure and theoretical TDDFT absorption of UVAG. Yellow, red, and small blue spheres represent, respectively, carbon, oxygen, and hydrogen atoms.

cell:

$$
\alpha(\lambda)=\sum_{i}^{\text {oxid.groups }} a_{i} \alpha_{i}^{\text {theor }}(\lambda),
$$

where $\alpha_{i}^{\text {theor }}(\lambda)$ are the absorption coefficients of each single oxidized group and $a_{i}$ are the linear combination coefficients. This approach is possible because of the direct proportionality between the intensity of the optical absorption spectra calculated below $4.96 \mathrm{eV}$ and the concentration of oxidized groups. Thanks to this proportionality, demonstrated by several tests $[8,20,21]$, we could perform calculations with one oxidized group per unitary cell. In fact, the optical spectra obtained by reducing the level of oxidation (considering one oxidized group every two and four cells) gives spectra with the same shape. Only the intensity changes proportionally to the oxidized groups concentration.

We used a minimal square algorithm to determine the linear combination coefficients that minimize the difference between experimental and theoretical spectra. The fitting procedure has been applied in the optical absorption range going from 1.18 to $4.96 \mathrm{eV}$. The coefficients obtained through this procedure are proportional to the relative concentration of each oxidized group included in our model. Despite that TDDFT is an exact theory, its actual application requires some approximations in the evaluation of the kernel, which includes the exchange-correlation interaction acting between electrons in the Casida algorithm for the calculation of the optical absorption spectrum. We performed some test calculations of the relative concentrations of oxidized groups in sample TA by using several different local adiabatic approximations (LDA [40], PBE [41], BLYP [35]) in order to evaluate the error induced by the approximated evaluation of the kernel. The change in the relative concentrations resulting from the use of these kernels is estimated to be below $10 \%$. This procedure allows to obtain chemical information from nondestructive optical reflectance measurements.

In order to evaluate the number of moles of oxidized groups per gram of cellulose, we used the property observed in Refs. [8,20,21] that the intensity of the experimental optical absorption $\alpha(\lambda)$ is proportional to the number of oxidized groups $N_{\text {ox/mole }}$ contained in a mole of cellulose: $\alpha(\lambda)=\alpha_{0}(\lambda) N_{\mathrm{ox} / \text { mole }}$, where $\alpha_{0}(\lambda)$ is the absorption of a sample with only one oxidized group per mole of cellulose. Theoretical absorption spectra can be normalized in such a way to obtain spectra of virtual cellulose samples with one oxidized group per cell, and that contain therefore an Avogadro number $\left(N_{A}\right)$ of oxidized groups per mole of cellulose: $\alpha(\lambda)_{\text {norm }}^{\text {theor }}=\alpha_{0}(\lambda) N_{A}$. Consequently, for any value of $\lambda$, the number of moles of oxidized groups per gram of cellulose $\left(n_{g}\right)$ is proportional to the ratio of the integral of experimental over the integral of the theoretical absorption spectra:

$$
n_{g}=\frac{N_{\mathrm{ox} / \mathrm{mole}}}{M}=\frac{\int \alpha(\lambda) d \lambda}{M \int \alpha(\lambda)^{\mathrm{theor}} d \lambda},
$$

where $M$ is the mass of a mole of cellulose (324 g). By multiplying $n_{g}$ by the value of the relative concentrations of oxidized groups obtained by the procedure described above, and by normalizing them according to the volume fraction $v$ of cellulose fibers, we obtain their concentrations.

\section{RESULTS AND DISCUSSION}

We applied our method to the ancient paper samples (38_C3 and 70L15), and to the modern paper sample (TA) used in industrial applications as an insulator for electrical transformers. In Fig. 7, we show the experimental and theoretical absorption coefficient of all the samples studied. The agreement between experiment and theory is excellent for ancient samples and good for TA sample. This validates our theoretical model, which is based on a perfect cellulose crystal with moderate oxidation of $\beta$-D-glucopyranose units typical of aging at room temperature. Furthermore, the amorphous regions of cellulose fibers in ancient paper tends to be consumed leaving mostly crystalline cellulose, which are better described in our model. In addition, it seems that small percentage of gelatin eventually present in ancient paper can be neglected in the calculations.

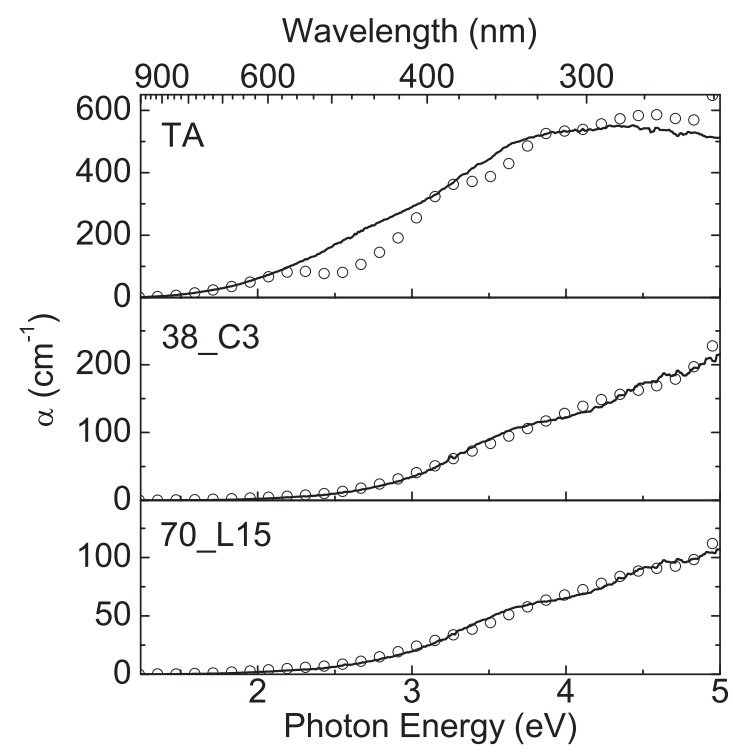

FIG. 7. Experimental (lines) and theoretical (symbols) absorption spectra of samples TA, 38_C3, and 70_L15. 

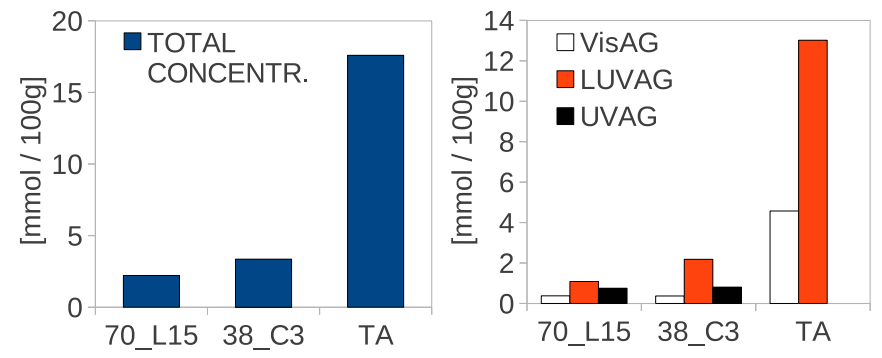

FIG. 8. (Color online) (Left) For each sample, total concentration of chromophores in moles per 100 grams of cellulose. (Right) For each sample, concentration of VisAG, LUVAG, and UVAG chromophores in moles per 100 grams of cellulose.

Transformer paper, on the other hand, even if composed of pure cellulose fibers, has been aged in very harsh conditions, at a temperature around $150^{\circ} \mathrm{C}$. Above that temperature, degradation phenomena of cellulose polymers could result in the ring opening of $\beta$-D-glucopyranose units followed by oxidation [42]. As a consequence, the formation of a larger set of different acidic products, such as simple carboxylic, dicarboxylic, and hydroxy-carboxylic acids, as well as hydroxybenzoquinones, is expected $[19,43]$. These compounds, not considered in our present model, could have optical absorption in the Vis and UV range. This could explain why, for sample $\mathrm{TA}$, the agreement between theory and experiment is still good but not excellent as for ancient paper samples. We can suppose that some degradation compounds could act as chromophores mainly absorbing around $2.7 \mathrm{eV}$ (about $460 \mathrm{~nm}$ ) (see Fig. 7). The theoretical model allows to evaluate the relative concentrations of VisAG, LUVAG, and UVAG present in the sample and their concentrations.

Despite that samples 38_C3 and 70_L15 are much older than TA, this last shows a level of oxidation one order of magnitude larger than the first two samples (see Fig. 8). The total concentrations for samples 38_C3, 70_L15, and TA are, respectively, 3.36, 2.21, and 17.59 mmoles per $100 \mathrm{~g}$ of cellulose. This indicates that high temperature affecting TA sample inside an electrical transformer in few years had a much more degrading effect than environmental degradation factors that affected ancient paper samples during several centuries. It would be interesting to deeply analyze this aspect in future works in order to use our model for tracking degradation of paper insulation, which is an important topic for power transmission industry.

The analysis of the relative concentrations of chromophores (see Fig. 9) shows that the TA sample is characterized by the highest VisAG and negligible UVAG relative concentrations. This indicates that the optical degradation of TA is more relevant with respect to the other two samples, not only because of the larger concentration of chromophores present in the sample but also for their character, since VisAG and LUVAG are the chromophores that create more visual damage in paper $[8,20,21]$.

Considering the relative concentrations of the three samples, we observe that the LUVAG relative concentration has a tendency to increase in the most degraded samples (those with the highest concentration of chromophores) further increasing

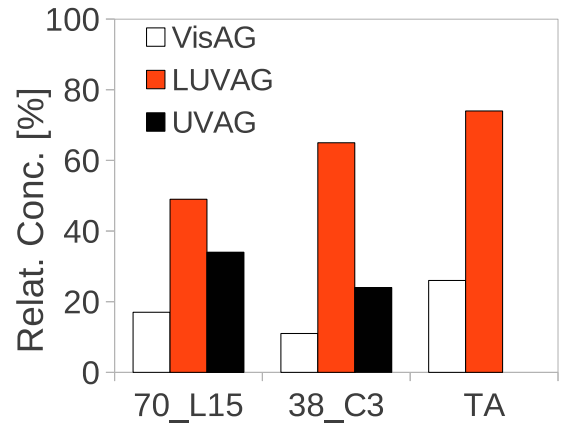

FIG. 9. (Color online) Relative concentrations of VisAG, LUVAG, and UVAG in samples 38_C3, 70_L15, and TA.

the visual damage, while the UVAG relative concentration has an opposite trend.

\section{CONCLUSION}

In the present study, we extended the Y-M model for the optical response of inhomogeneous materials to optically thick samples. An expression for the scattering coefficient of light [Eq. (16)] was calculated in this regime and its validity for strongly absorbing paper samples was demonstrated. In this way, the cellulose absorption coefficient were recovered up to the ultraviolet region from nondestructive reflectance measurements. Furthermore, $a b$ initio TDDFT simulations were applied to fit the experimental absorption and extract chemical information at the molecular scale. By using this approach, the concentration of oxidized groups acting as chromophores in two ancient paper samples and in a modern paper sample used in industrial applications as an insulator in electrical transformers were evaluated.

We found that the relative concentration of different oxidized groups in cellulose depends on the aging temperature endured by samples. This clearly indicates that the oxidation of cellulose follows different reaction pathways depending on temperature. This aspect is worth further investigation to shed light into the degradation mechanisms of paper and cellulose fibers at different aging temperatures. Our approach has a wide range of applications for cellulose-based materials, like paper, textiles, and other manufactured products of great industrial and cultural interest. It can also be extended to other strongly absorbing inhomogeneous materials.

\section{ACKNOWLEDGMENTS}

All authors thank the Centro Italiano di Studi e Ricerche di Storia e Tecnologia della Carta "Andrea F. Gasparinetti" (Fabriano, Italy) and the Istituto Centrale per il Restauro e la Conservazione del Patrimonio Archivistico e Librario (Roma, Italy) for their support. Energopomiar Elektryka sp.z o.o. in Gliwice (Poland) is thanked for providing the transformer paper samples used in this study. We also acknowledge CPU time granted by CINECA, and funding from the EC's FP7 Grants No. 211956 (ETSF user project 211). 
[1] H. Moosmueller, R. K. Chakrabarty, and W. P. Arnott, J. Quant. Spectrosc. Radiat. Transfer 110, 844 (2009).

[2] Y. Shkuratov, V. Kaydash, V. Korokhin, Y. Velikodsky, N. Opanasenko, and G. Videen, Planet. Space Sci. 59, 1326 (2011).

[3] S. L. Jacques, Phys. Med. Biol. 58, R37 (2013).

[4] A. B. Murphy, Sol. Energ. Mat. Sol. Cells 91, 1326 (2007).

[5] M. Elias, L. Simonot, M. Thoury, and J.-M. Frigerio, Opt. Commun. 231, 25 (2004).

[6] C. Clementi, C. Miliani, G. Verri, S. Sotiropoulou, A. Romani, B. G. Brunetti, and A. Sgamellotti, Appl. Spectosc. 63, 1323 (2009).

[7] M. Missori, M. Righini, and S. Selci, Opt. Commun. 231, 99 (2004).

[8] A. Mosca Conte, O. Pulci, A. Knapik, J. Bagniuk, R. Del Sole, J. Lojewska, and M. Missori, Phys. Rev. Lett. 108, 158301 (2012).

[9] M. Kerker, The Scattering of Light and Other Electromagnetic Radiation (Academic, New York, 1969).

[10] G. Kortum, Reflectance Spectroscopy (Principles, Methods, Applications) (Springer-Verlag, Berlin-Heidelberg-New York, 1969).

[11] M. Missori, C. Mondelli, M. De Spirito, C. Castellano, M. Bicchieri, R. Schweins, G. Arcovito, M. Papi, and A. C. Castellano, Phys. Rev. Lett. 97, 238001 (2006).

[12] M. De Spirito, M. Missori, M. Papi, G. Maulucci, J. Teixeira, C. Castellano, and G. Arcovito, Phys. Rev. E 77, 041801 (2008).

[13] D. Hunter, Papermaking: The History and Technique of an Ancient Craft (Dover, New York, 1978).

[14] M. Missori, M. Righini, and A. L. Dupont, Opt. Commun. 263, 289 (2006).

[15] N. Pauler, Paper Optics (AB Lorentzen \& Wettre, Kista, Sweden, 2002).

[16] D. Klemm, B. Philipp, T. Heinze, U. Heinze, and W. Wagenknecht, Comprehensive Cellulose Chemistry; Volume I: Fundamentals and Analytical Methods (WILEY-VCH Verlag GmbH: Weinheim, Germany, 1998).

[17] T. Lojewski, P. Miskowiec, M. Missori, A. Lubanska, L. M. Proniewicz, and J. Lojewska, Carbohydr. Polym. 82, 370 (2010).

[18] S. Zervos, Natural and Accelerated Ageing of Cellulose and Paper: A Literature Review, edited by A. Lejeune and T. Deprez (Nova, New York, 2010), pp. 155-203.

[19] C. Corsaro, D. Mallamace, J. Lojewska, F. Mallamace, L. Pietronero, and M. Missori, Sci. Rep. 3, 2896 (2013).
[20] A. Mosca Conte, O. Pulci, R. Del Sole, A. Knapik, J. Bagniuk, J. Lojewska, L. Teodonio, and M. Missori, e-J. Surf. Sci. Nanotech. 10, 569 (2012).

[21] A. Mosca Conte, C. Violante, M. Missori, F. Bechstedt, L. Teodonio, E. Ippoliti, P. Carloni, L. Guidoni, and O. Pulci, J. Electron Spectr. Rel. Phenom. 189S, 46 (2013).

[22] P. Kubelka, J. Opt. Soc. Am. 38, 448 (1948).

[23] A. M. Scallan and J. Borch, TAPPI J. 57, 143 (1974).

[24] L. Yang and S. J. Miklavcic, J. Opt. Soc. Am. A 22, 1866 (2005).

[25] L. Yang and S. J. Miklavcic, Opt. Lett. 30, 792 (2005).

[26] B. Philips-Invernizzi, D. Dupont, and C. Caz, Opt. Eng. 40, 1082 (2001).

[27] R.-J. Roe, Methods of X-Ray and Neutron Scattering in Polymer Science (Oxford University Press, New York, 2000).

[28] M. Missori, M. De Spirito, L. Ferrari, S. Selci, A. Gnoli, F. R. Bertani, A. S. Girgis, H. El-Saied, and A. H. Basta, J. Nanopart. Res. 14, 649 (2012).

[29] E. Runge and E. K. U. Gross, Phys. Rev. Lett. 52, 997 (1984).

[30] D. Varsano, A. Marini, and A. Rubio, Phys. Rev. Lett. 101, 133002 (2008).

[31] O. Pulci, A. Marini, M. Palummo, and R. Del Sole, Phys. Rev. B 82, 205319 (2010).

[32] H. A. Krassig, Cellulose (Gordon and Breach Science Publisher, Amsterdam, The Netherlands, 1996), p. 27.

[33] A. C. O'Sullivan, Cellulose 4, 173 (1997).

[34] P. Hohenberg and W. Kohn, Phys. Rev. 136, B864 (1964).

[35] C. Lee, W. Yang, and R. G. Parr, Phys. Rev. B 37, 785 (1988).

[36] http://www.quantum-espresso.org

[37] M. E. Casida, Recent Developments and Applications of Modern Density Functional Theory, Theoretical and Computational Chemistry Vol. 4 (J. M. Seminario, Elsevier, Amsterdam, 1996).

[38] M. A. L. Marques, C. A. Ullrich, F. Nogueira, A. Rubio, K. Burke, and E. K. U. Gross, Time-Dependent Density Functional Theory (Springer-Verlag, Berlin, Heidelberg, Germany, 2006).

[39] http://dp-code.org

[40] J. P. Perdew and A. Zunger, Phys. Rev. B 23, 5048 (1981).

[41] J. P. Perdew, K. Burke, and M. Ernzerhof, Phys. Rev. Lett. 77, 3865 (1996).

[42] A. J. Michell and H. G. Higgins, Infrared Spectroscopy in Australian Forest Products Research (CSIRO Forestry and Forest Products, Melbourne, Australia, 2002).

[43] T. Rosenau, A. Potthast, K. Krainz, Y. Yoneda, T. Dietz, Z. Peralta-Inga Shields, and A. D. French, Cellulose 18, 1623 (2011). 